\title{
Colorectal cancer and adenoma screening using urinary volatile organic compound (VOC) detection: early results from a single-centre bowel screening population (UK BCSP)
}

\author{
E. Mozdiak ${ }^{1}$ (1) A. N. Wicaksono ${ }^{2} \cdot$ J. A. Covington ${ }^{2} \cdot$ R. P. Arasaradnam ${ }^{1}$
}

Received: 1 September 2018 / Accepted: 8 March 2019 / Published online: 15 April 2019

(c) The Author(s) 2019

\begin{abstract}
Background The United Kingdom (UK) bowel cancer screening programme has reduced mortality from colorectal cancer (CRC), but poor uptake with stool-based tests and lack of specificity of faecal occult blood testing (FOBT), has prompted investigation for a more suitable screening test. The aim of this study was to investigate the feasibility of a urinary volatile organic compounds (VOC)-based screening tool for CRC.

Methods The urine from FOBT-positive patients was analysed using field asymmetric ion mobility spectrometry (FAIMS) and gas chromatography coupled with ion mobility spectrometry (GC-IMS). Data were analysed using a machine learning algorithm to calculate the test accuracy for correct classification of CRC against adenomas and other gastrointestinal pathology.

Results One hundred and sixty-three patients were enrolled in the study. Test accuracy was high for differentiating CRC from control: area under the curve (AUC) 0.98 (95\% CI 0.93-1) and 0.82 (95\% CI 0.67-0.97) using FAIMS and GC-IMS respectively. Correct classification of CRC from adenoma was high with AUC range 0.83-0.92 (95\% CI 0.43-1.0). Classification of adenoma from control was poor with AUC range 0.54-0.61 (95\% CI 0.47-0.75) using both analytical modalities. Conclusions CRC was correctly distinguished from adenomas or no bowel pathology using urinary VOC markers, within the bowel screening population. This pilot study demonstrates the potential of this method for CRC detection, with higher test uptake and superior sensitivity than FOBT. In addition, this is the first application of GC-IMS in CRC detection which has shown high test accuracy and usability.
\end{abstract}

Keywords Colorectal cancer · Bowel cancer screening · Volatile organic compounds · Urinary biomarkers

\section{Introduction}

Colorectal cancer (CRC) is a leading cause of cancer-related deaths in the western world [1, 2]. It represents the fourth most common cancer in England and Wales and is the second leading cause of cancer-related deaths [1].

Electronic supplementary material The online version of this article (https://doi.org/10.1007/s10151-019-01963-6) contains supplementary material, which is available to authorized users.

E. Mozdiak

ella.mozdiak@nhs.net

1 University Hospitals Coventry and Warwickshire, Coventry, UK

2 School of Engineering, The University of Warwick, Coventry, UK
The United Kingdom (UK) bowel cancer screening programme (UK BCSP) was implemented in 2007 following a series of randomised controlled trials that demonstrated a reduction in mortality from CRC due to screening [3-6]. However, the current guaiac FOBT has a number of well-documented disadvantages including low uptake (around 60\%), low specificity and the test interpretation has the potential for human error $[7,8]$. The introduction of the faecal immunochemical test (FIT) as the first-line bowel screening test in the UK is anticipated within the next year and is associated with improved uptake by as much as $25 \%$ and higher sensitivity [7, 9]. However, overall test accuracy could be improved further, to detect more CRC cases via screening and prevent unnecessary colonoscopies. 
There is a dearth of research on new biomarkers for $\mathrm{CRC}$ detection. One area that has gained momentum over the past decade is the use of volatile organic compounds (VOCs). VOCs are organic chemicals that have a high vapour pressure at room temperature, i.e. that evaporate or sublimate readily under ambient conditions. They can be captured from a variety of body mediums and have been shown to alter in different disease states $[10,11]$.

Applying the use of gas phase markers to diagnose CRC is a rapidly expanding area, but it involves highly complex sample and data analysis. Detection of VOCs in CRC using a variety of different mediums has been investigated in small-scale studies [12-14]. Few have used urine analysis or focussed on the asymptomatic BCSP cohort. There are currently no VOC-based tests established in the clinical setting for any disease [15-18].

Using urine for VOC detection has the advantage that urine is simple to collect and is readily available and collection is associated with high patient acceptability. It is also easy to store and shows stability in the medium term [19]. These factors make it an ideal focus for the development of a screening tool as an alternative to a faeces-based test.

This study aims to establish whether urinary VOC markers can be utilised as a tool for detecting CRC and adenomas within the UK BCSP. Sample analysis was conducted using field asymmetrical ion mobility spectrometry (FAIMS), which has been employed in a small number of studies before [20-23]. In addition, a new analytical modality for disease detection called gas chromatography coupled with ion mobility spectrometry (GC-IMS) was employed.

\section{Materials and methods}

All enrolled patients were recruited from the Coventry and Warwickshire University Hospitals between April 2015 and November 2016. Regional ethical approval was granted by the Warwickshire Research and Development Department and Warwickshire Ethics Committee 09/ H1211/38. Informed consent was obtained from the individual participants that took part in the study. This study was approved by the bowel cancer screening research committee only to approach those that had a positive FOBT test.

\section{Patient recruitment}

Patients were recruited from the nurse-led BCSP clinics following a positive FOBT result. Consent and urine sample collection were carried out at the clinic prior to bowel preparation administration. A total of 181 patients were invited to participate and 163 consented to provide samples for the final analysis.

\section{Sample collection and storage}

Two $20 \mathrm{ml}$ samples of urine were collected. Samples were immediately transferred to $-20{ }^{\circ} \mathrm{C}$ storage and then to $-80{ }^{\circ} \mathrm{C}$ within $24 \mathrm{~h}$ for long-term storage. Diagnostic outcome data were collected from the colonoscopy or computed tomography (CT) colonography result, histology was confirmed from the pathology report.

\section{Sample analysis}

Samples were analysed using the Lonestar FAIMS instrument (Owlstone, UK) and the Silox GC-IMS (Imspex, UK). FAIMS is a gas detection technology that separates chemical ions, within a complex mixture of VOCs, based on their mobility/movement in high electrical fields. This technique has been described in detail before. Sampling methods can be found in previous publications by our group [12, 23]. A detailed schematic for FAIMS analysis is found in Online Resource 1.

GC-IMS involves a two-stage analytical process. The first stage uses a gas chromatograph to separate VOCs based on their interaction with a coated capillary column. Then these VOCs are detected as they elude from the column using a drift-tube ion mobility spectrometer, where the time taken for chemical ions to travel along a tube (against a flow of buffer gas) is measured. Chemical ions of different sizes take different lengths of time to travel along the tube and this can be used to separate chemical species. As this has 
Fig. 1 Schematic of the separation process and ion detection using gas chromatographyion mobility spectrometry (GC-IMS). (1) Sample passes through the gas column where initial separation occurs. (2) The discrete compounds are consecutively fed into the ionisation chamber where ionisation occurs. (3) Ions pass through the drift tube at varying speeds dependent on their mobility. (4) Ions hit the sensor plate and are detected. (5) Ion peaks are calculated based on drift time
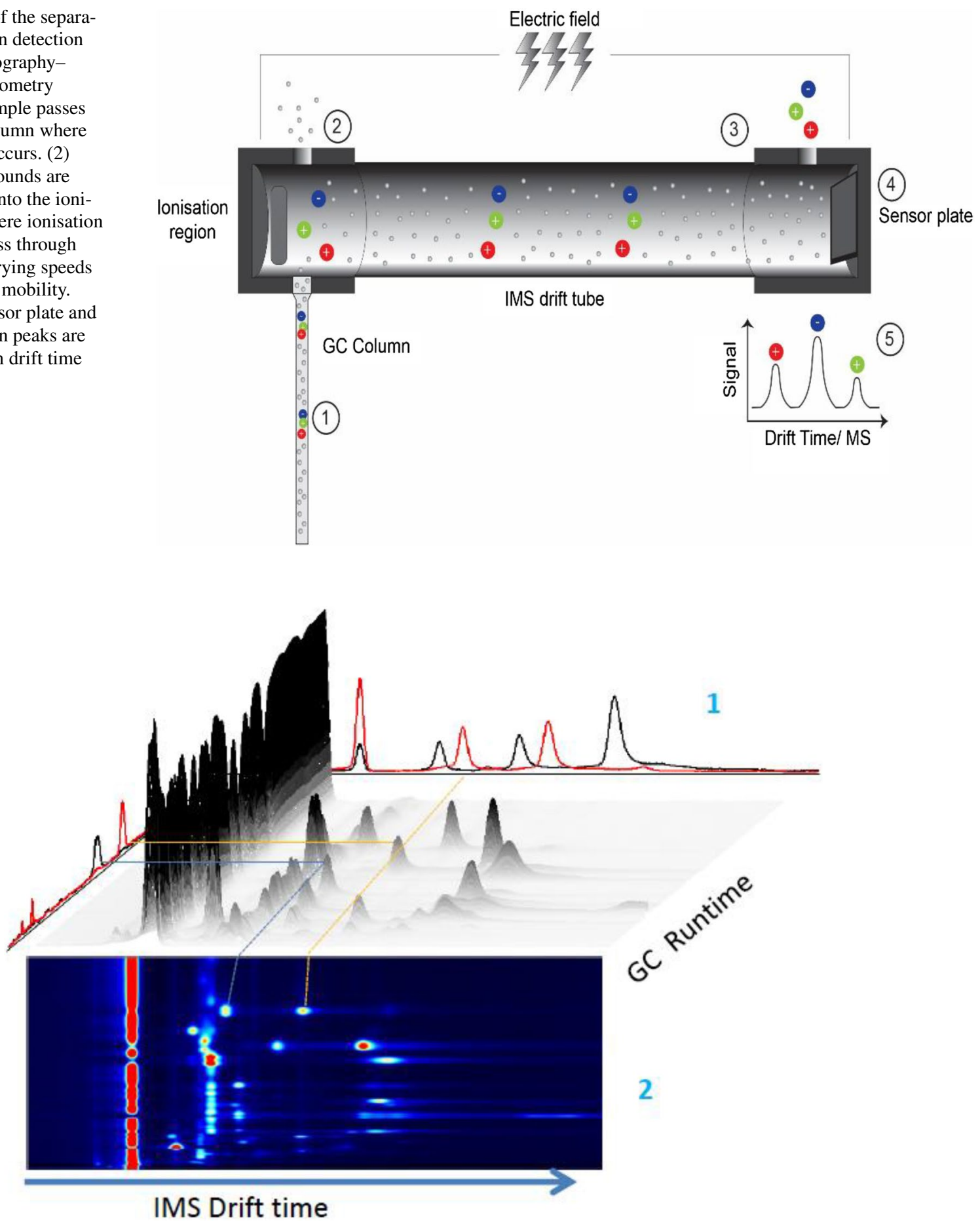

Fig. 2 Three-dimensional representation of gas chromatography (GC) data output with corresponding ion mobility spectrometry (IMS) chromatogram. (1). Single IMS spectra data is combined with GC run time peaks. (2) Heatmap corresponding to GC-IMS peaks (yellow and blue lines) (Image adapted with permission from Impsex, UK). Data output is twofold: gas chromatography (GC) gives peaks representing retention time as the ions pass thorough the column. This is coupled with ion mobility spectrometry (IMS) data, based on the mobility of the ions as they pass through the drift tube and hit the sensor. The culmination of this two-phase analysis is represented as an IMS chromatogram which incorporates millions of data points in a heatmap (Fig. 2). These data points are subject to very similar statistical analysis as is applied to the Lonestar data 
not previously been described sample analysis is outlined here:

A $5 \mathrm{ml}$ urine sample is aliquoted into a $20 \mathrm{ml}$ glass vial and sealed with a crimp lid. A $21 \mathrm{~g}$ needle is attached to the GC-IMS input port. The needle with attached port is inserted into the sample headspace $1 \mathrm{~cm}$ above the urine. The needle is held in place for $20 \mathrm{~s}$ to allow for vapour aspiration. The total run time is 5 min per sample. The carrier gas flow rate is $150 \mathrm{ml} / \mathrm{min}$ and sample flow rate through the instrument is $20 \mathrm{ml} / \mathrm{min}$. The sample heating was carried out in accordance with manufacturer instructions to a maximal level of $80{ }^{\circ} \mathrm{C}$. A schematic for the GC-IMS detection process is shown in Fig. 1 and the three-dimensional data output with corresponding heat map is shown in Fig. 2.

\section{Statistical analysis}

The analysis of data created through FAIMS and GC-IMS analysis employs machine learning methods. In brief, they involve the construction of computerised algorithms that can learn from and make predictions on the output data from the instrument. The algorithms build a prediction model from a training set of known cases and use this knowledge to predict output decisions (diagnoses) on unknown cases. Five classification models were used; each dataset was compared with each model to find the most accurate for each specific set of samples. This form of analysis produces receiver operating characteristic (ROC) curves with area under the curve (AUC), sensitivity and specificity values calculated from the coordinates of the ROC plots. A schematic of the analysis pipeline is provided in Online Resource 2. All statistical analyses were carried out using the verification package in R studio (R Foundation for Statistical Computing, Vienna, Austria).

As CRC numbers were small within the screening population (incidence of 8-10\%), a balancing technique was applied to the data to fairly match the non-CRC samples with the same number of CRC samples and avoid bias from an unbalanced control group. Balancing involved the well-described synthetic minority over-sampling technique (SMOTE), where artificially generated points are plotted to represent the control group as a whole and is used to provide a more fair representation [24].

\section{Results}

\section{FAIMS analysis}

A total of 163 samples were analysed. 93 (57\%) were from males, median age of patients was 67 years, $12(7.4 \%)$ were current smokers. 41 (25.4\%) were ex-smokers and 109 $(67.2 \%)$ had never smoked. Patients were grouped into categories according to diagnosis for analysis. Diagnostic outcomes for study participants are listed in Table 1.

Group (a) CRC vs normal control demonstrated the highest degree of separation with AUC 0.98 (95\% CI 0.93-1.0) with 12 patients in each group. The corresponding ROC curve is shown in Fig. 3. Sensitivity and specificity were also high: 1.0 (95\% CI 0.74-1) and 0.92 (95\% 0.62-1), respectively (Table 2).

Table 1 Diagnostic outcomes for study participants and distribution of CRC by site (total of 13 cancer sites as one patient had a synchronous tumours)

\begin{tabular}{lll}
\hline Diagnosis & Number (\%) & \\
\hline Cancer & Total & $12(7.6)$ \\
& Rectum & $4(2.4)$ \\
& Sigmoid & $4(2.4)$ \\
& Tescending colon & 0 \\
& Ascending colon & $1(0.58)$ \\
& Cecum & $2(1.17)$ \\
Adenoma & Total & $2(1.17)$ \\
& High & $80(49.1)$ \\
& Intermediate & $17(10.5)$ \\
Diverticular disease & Low & $36(21.1)$ \\
Normal & & $27(17.5)$ \\
Haemorrhoids & & $14(8.2)$ \\
Other & & $37(19.3)$ \\
Excluded & & $5(2.9)$ \\
*1 not fit enough for investigations, 7 declined investigations & $14(8.2)^{\wedge}$ \\
Inflammatory bowel disease: $n=7$, rectal telangiectasia: & $n=2$, rec- \\
tal ulcer: $n=1$, radiation proctitis: $n=1$, inflammatory pseudopolyp: \\
$n=1$, non-specific sigmoid inflammation: $n=1$, ischaemic sigmoid \\
stricture: $n=1$ & & $8(4.7)^{*}$ \\
\hline
\end{tabular}


Table 2 Classification of BCSP study participants by outcome using FAIMS

\begin{tabular}{llcccc}
\hline Group & AUC & Sensitivity & Specificity & PPV & NPV \\
\hline a) CRC (12) vs normal (12) & $0.98(0.93-1)$ & $1(0.74-1)$ & $0.92(0.62-1)$ & 0.92 & 1 \\
b) CRC + all adenomas (93) vs normal (37) & $0.64(0.54-0.74)$ & $0.48(0.38-0.59)$ & $0.89(0.75-0.97)$ & 0.92 & 0.41 \\
c) CRC + high-risk adenomas (30) vs normal (37) & $0.62(0.48-0.76)$ & $0.57(0.37-0.75)$ & $0.68(0.5-0.82)$ & 0.59 & 0.66 \\
d) CRC + high-risk adenomas (30) vs other (70) & $0.6(0.47-0.73)$ & $0.47(0.28-0.66)$ & $0.80(0.68-0.89)$ & 0.52 & 0.76 \\
e) CRC + all adenomas (93)vs other (70) & $0.56(0.47-0.65)$ & $0.91(0.84-0.96)$ & $0.25(0.15-0.38)$ & 0.64 & 0.67 \\
f) Non-CRC (113) vs normal (37) & $0.61(0.51-0.71)$ & $0.56(0.46-0.65)$ & $0.68(0.5-0.82)$ & 0.83 & 0.35 \\
g) CRC (12) vs Adenoma (7) (hr) & $0.92(0.77-1)$ & $0.83(0.52-0.98)$ & $1(0.59-1)$ & 1 & 0.78 \\
h) CRC (12) vs Adenoma (12) (ir) & $0.84(0.67-1)$ & $0.83(0.52-0.98)$ & $0.75(0.43-0.95)$ & 0.77 & 0.82 \\
i) CRC (12) vs Adenoma (12) (1r) & $0.83(0.66-1)$ & $0.75(0.43-0.95)$ & $0.92(0.62-1)$ & 0.90 & 0.79 \\
\hline
\end{tabular}

Using sparse logistic regression and Gaussian process

Corresponding 95\% CIs are stated in brackets. Numbers in brackets in group column denote sample number

$B S C P$ bowel cancer screening programme, FAIMS field asymmetric waveform ion mobility spectrometry, CRC Colorectal cancer

\section{SparseLogisticRegression $($ AUC $=0.98)$}

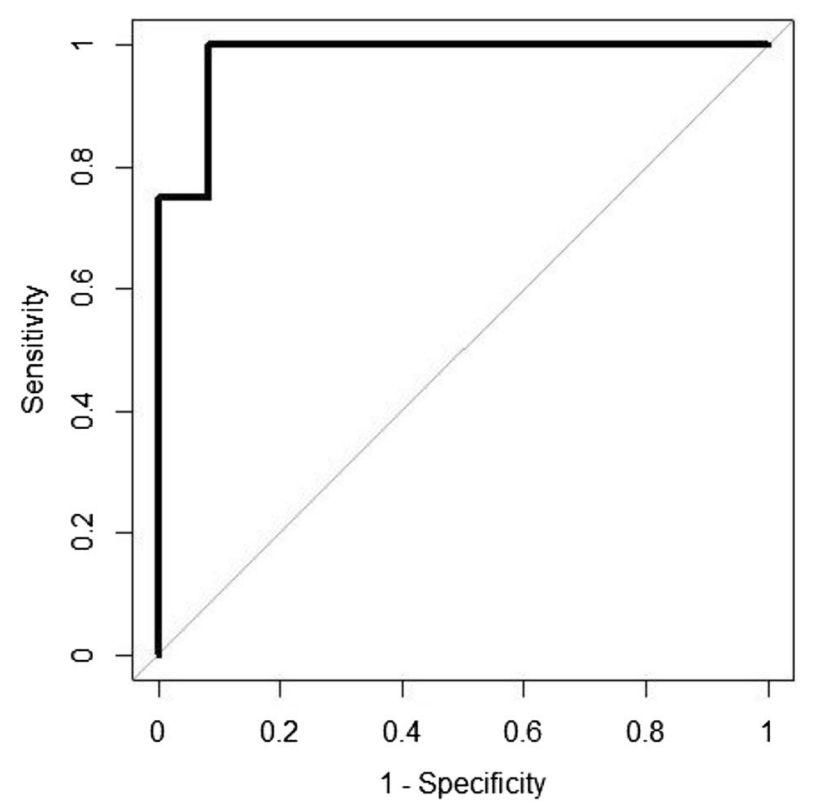

Fig. 3 Receiver operating characteristic (ROC) curve for classification of colorectal cancer (CRC) vs normal in bowel cancer screening programme (BCSP) patients (balanced) using the sparse logistic regression classifier using field asymmetric waveform ion mobility spectrometry(FAIMS)

In groups (b-e) CRC was grouped with adenomas and showed only modest AUC, sensitivity and specificity results, when attempting to classify groups according to diagnosis. The most accurate classification of the adenoma groups was seen in (b) CRC + all adenomas vs normal control; here sensitivity was low at $0.48(95 \%$ CI $0.38-0.59)$ but specificity was high at 0.89 (95\% CI 0.75-0.97). In (f) when CRC was excluded, separation was low with sensitivity 0.56 (95\% CI $0.46-0.65$ ).

A further set of analyses were carried out to investigate the classification of the adenoma groups in more depth and to compare CRC with three categories of adenoma: $(\mathrm{g})$ high risk, (h) intermediate risk and (i) low risk, according to the BSG guidelines [25], the results are displayed in Table 1. High sensitivity was demonstrated when each adenoma group was compared with CRC. The most accurate overall classification was seen in CRC vs high-risk adenoma with a sensitivity of 0.83 (95\% CI $0.52-0.98$ ) and specificity of 1 (95\% CI 0.59-1).

\section{GC-IMS analysis}

One hundred and nine patient samples were analysed using the Silox GC-IMS instrument. Five comparator groups were devised according to outcome (Table 3).

As with the analysis using FAIMS, when comparing CRC vs normal control (group a) there was a high degree of separation with a sensitivity of 0.80 (95\% CI 0.44-0.97) and specificity of 0.83 (95\% CI 0.63-0.95). The corresponding ROC curve is seen in Fig. 4. CRC vs other diagnoses also had a high sensitivity of 1.0 (95\% CI $0.66-1)$, however, specificity dropped to 0.57 (95\% CI 0.34-0.78). When CRC samples were grouped with adenomas and compared with other groups (those with any diagnosis other than CRC or adenoma) the sensitivity dropped to a modest level of 0.71 (95\% CI $0.58-0.81)$ with sensitivity 0.55 (0.39-0.70). Adenomas vs normal control showed a low level of separation, with a sensitivity of only 0.58 (95\% CI $0.44-0.71$ ) and specificity 0.62 (95\% CI 0.41-0.81) (Table 3). 


\section{GaussianProcess ( AUC $=0.82$ )}

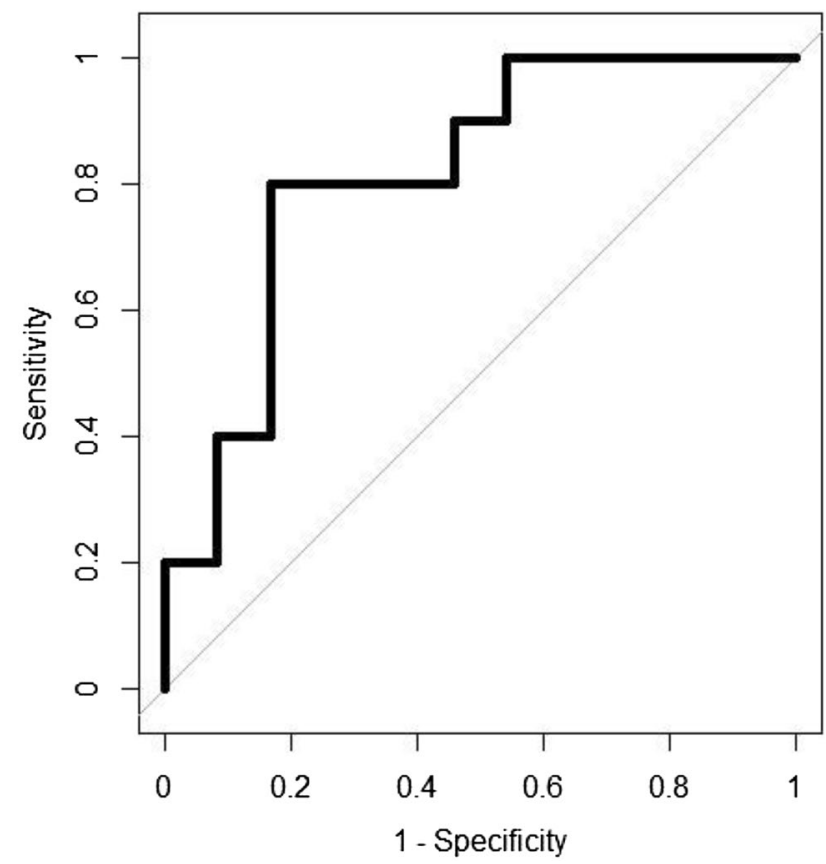

Fig. 4 Receiver operating characteristic (ROC) curve for classification of colorectal cancer (CRC) vs normal using GC-IMS. [Gaussian process (GP) classifier]

\section{Discussion}

This study explored the feasibility of a urinary VOCbased test in the detection of CRC within the screening population that tested positive for faecal occult blood. The results, applying two different technologies-FAIMS and GC-IMS show consistency. Ability to distinguish CRC from normal control was high with AUC 0.98, sensitivity of 1.0 and specificity of 0.92 . Ability to distinguish CRC from low-, intermediate- and high-risk adenomas was high with test accuracy ranging from 0.83 to 0.92 using FAIMS.
Similarly, ability to distinguish CRC from normal control was high with AUC 0.82 , sensitivity of 0.80 and specificity of 0.83 using GC-IMS.

CRC-specific VOCs are thought to occur via genetic and protein changes that cause peroxidation of the cell membrane [26]. In addition, there is an increase in reactive oxygen species within the cancer cell and alterations in the microbiome have a direct effect on the VOCs [27-30]. How distinct these changes are in the CRC group compared with other disease groups is yet to be fully elucidated.

The separation of CRC from normal controls was high, yet when CRC cases were grouped with adenomas the accuracy dropped significantly. This suggests that CRC has a unique VOC profile or signature that distinguishes it from other gastrointestinal pathologies. When this profile is combined with other (non-neoplastic) gastrointestinal disorders, the VOC signature is not sufficiently distinct to allow correct classification.

In the case of CRC vs different adenoma risk groups, the separation was again high. This implies that it is possible to separate malignant from pre-malignant disease based on urinary VOC signature patterns. When considering this as a basis for a screening test, the high specificity suggests the potential of using VOC-based analysis to reduce the number of unnecessary endoscopic procedures. This set of results warrant further exploration, to ascertain whether the separation seen is simply because the adenoma group represents another non-CRC group or whether there are changes specific to the VOC signature of colonic adenomas that make it more distinct from CRC than other groups.

To the best of our knowledge this is the first study to specifically examine adenoma detection by urinary VOCs. Applying FAIMS, the adenoma group showed poor separation from normal controls. Previous studies have reported on colonic adenoma detection by faecal [31] VOCs with low to modest test accuracy (sensitivity of 0.62 ). Advanced adenoma detection using breath VOCs [32,33] demonstrated more encouraging results (sensitivity 1.0). These conflicting results suggest that there is more work needed to establish the mechanism of VOC signature changes in the presence

Table 3 Classification of BCSP study participants using GC-IMS using Gaussian process or support vector machine

\begin{tabular}{|c|c|c|c|c|c|}
\hline Group & AUC & Sensitivity & Specificity & PPV & NPV \\
\hline CRC (10) vs normal (24) & $0.82(0.67-0.97)$ & $0.80(0.44-0.97)$ & $0.83(0.63-0.95)$ & 0.67 & 0.91 \\
\hline $\mathrm{CRC}+$ high-risk adenomas (23) vs normal(24) & $0.53(0.36-0.70)$ & $0.48(0.27-0.69)$ & $0.67(0.45-0.84)$ & 0.58 & 0.57 \\
\hline CRC (10) vs other (20) & $0.77(0.60-0.94)$ & $1(0.66-1)$ & $0.57(0.34-0.78)$ & 0.5 & 1 \\
\hline $\mathrm{CRC}+$ all adenomas $(65)$ vs other $(42)$ & $0.61(0.49-0.72)$ & $0.71(0.58-0.81)$ & $0.55(0.39-0.70)$ & 0.71 & 0.55 \\
\hline All adenomas (55) vs normal (24) & $0.61(0.47-0.75)$ & $0.58(0.44-0.71)$ & $0.62(0.41-0.81)$ & 0.78 & 0.39 \\
\hline
\end{tabular}

Corresponding 95\% CI are in brackets. Study numbers are stated in the group column in brackets

$B S C P$ bowel cancer screening programme, $G C-I M S$ gas chromatography coupled with ion mobility spectrometry, $A U C$ area under the curve, $C R C$ colorectal cancer, $P P V$ positive predictive value, $N P V$ negative predictive value 
of colonic adenomas and other gastrointestinal disorders. This is vital as adenomas represent a pre-malignant process with adenoma detection intrinsically linked to CRC mortality [34-36]. Adenoma detection is particularly pertinent to the BCSP population given the high adenoma incidence of approximately $50 \%$, compared with around $15 \%$ in the average population [25, 37]. As faecal testing relies on the presence or absence of blood, it is poor at detecting adenomas, as most do not bleed [38]. If the patients with CRC and adenomas could be better identified using a urine test, this could revolutionise the screening process. Urine sampling has been demonstrated to be more acceptable to patients than faecal in the previous work by our group. In this study, recruitment was $>90 \%$, far exceeding the FOBT uptake of approximately $60 \%$.

The analytical technology associated with VOC detection is constantly being improved, with particular focus on refinement of data software and ion capture technique. The benefits of using FAIMS as the detection method of choice, have been described in the literature before [21], but the level of repeatability required for equipping the clinical setting with this technology has not yet been demonstrated.

This is the first reported study demonstrating the application of GC-IMS in CRC detection [39, 40]. GC-IMS have several advantages as a clinical tool. It is simple to use, meaning specialist training is not required. Also, it is portable, thus in theory, could be transported to the clinical area of need for instant analysis. Finally, due to the IMS component, it has the technology to isolate and identify the chemical compounds within the urine sample. This has the potential to broaden current understanding of CRC pathogenesis and also narrow the target window of chemicals that comprise the VOC profile in CRC. However, at this point the chemical National Institute of Standards and Technology (NIST) library required to perform this step is currently small.

It is important to highlight that the bowel screening patient group recruited for this study consisted of patients that had a positive FOBT. The authors recognise the importance of examining FOBT negative patients too when comparing a new screening tool with FOBT, but were constrained by the BCSP recommendations.

The current guaiac FOBT has poor selectivity for CRC, therefore, it would be interesting to repeat this experiment once the FIT is introduced for UK bowel screening. It has both superior sensitivity and specificity compared to the current FOBT. Combining VOC detection with FIT as a twostage test in the asymptomatic population that forms the basis of a decision-making algorithm for further investigations, is an area of interest that may hold the most potential in the field of VOC diagnostics in CRC [41], rather than the use of VOC detection as a stand alone test. Further research could also test this algorithm (FIT plus urinary VOC profile) in the symptomatic population, where risk stratification is extremely difficult based on symptoms alone.

A limitation of this study was the small sample size for the CRC group, but this reflects the nature of the screening population with low CRC detection rates of around $8 \%$. Machine learning algorithms that were used to analyse both sets of date always risk the possibility of overfitting of the data. This was minimised using a cross-validation technique and using two different technologies.

\section{Conclusions}

Our results indicate detection of CRC and adenomas through urinary VOCs within a screening population is feasible. CRC can be correctly classified from control and adenomas using FAIMS and GC-IMS, but the classification of adenomas from control was poor. This approach to disease detection faces multiple challenges, that reflect the complexity of human disease and it is likely biomarker-led disease detection will require a panel of markers rather than focus on one specific marker in the screening population.

Acknowledgements The authors would like to thank the bowel cancer screening nurses Carol Wheatley and Paula Voisey and the gastroenterology research nurses: Subie Wurie, Nicola O'Connell and Leighanne Burns who were all invaluable to patient recruitment. We would like to thank the patients who generously provided samples for this study.

\section{Compliance with ethical standards}

Conflict of interest The authors declare that they have no conflict of interest.

Ethical approval All procedures performed in studies involving human participants were in accordance with the ethical standards of the institutional and/or national research committee and with the 1964 Helsinki Declaration and its later amendments or comparable ethical standards.

Informed consent All patients signed consent forms for inclusion in the study.

Open Access This article is distributed under the terms of the Creative Commons Attribution 4.0 International License (http://creativeco mmons.org/licenses/by/4.0/), which permits unrestricted use, distribution, and reproduction in any medium, provided you give appropriate credit to the original author(s) and the source, provide a link to the Creative Commons license, and indicate if changes were made.

\section{References}

1. Office for National Statistics: Mortality Statistics-Deaths registered in England and Wales Published online 2014. http:// www.ons.gov.uk/ons/publications/all-releases.html?definition $=\mathrm{tcm} \% 3 \mathrm{~A} 77-27475$. Accessed 3 July 2018 
2. World Health Organisation(WHO). Leading cause of death in Europe: fact sheet Copenhagen: WHO Regional Office for Europe (2012). https://data.euro.who.int/hfadb]. Accessed 3 July 2018

3. Mandel JS, Bond JH, Church TR, Snover DC, Bradley GM, Schuman LM et al (1993) Reducing mortality from colorectal cancer by screening for fecal occult blood. Minnesota Colon Cancer Control Study. N Engl J Med 328(19):1365-1371

4. Kewenter J, Brevinge H, Engaras B, Haglind E, Ahren C (1994) Results of screening, rescreening, and follow-up in a prospective randomized study for detection of colorectal cancer by fecal occult blood testing. Results for 68,308 subjects. Scand J Gastroenterol 29(5):468-473

5. Kronborg O, Fenger C, Olsen J, Jorgensen OD, Sondergaard O (1996) Randomised study of screening for colorectal cancer with faecal-occult-blood test. Lancet 348(9040):1467-1471

6. Hardcastle JD, Chamberlain JO, Robinson MH, Moss SM, Amar SS, Balfour TW et al (1996) Randomised controlled trial of faecal-occult-blood screening for colorectal cancer. Lancet 348(9040):1472-1477

7. Tinmouth J, Lansdorp-Vogelaar I, Allison JE (2015) Faecal immunochemical tests versus guaiac faecal occult blood tests: what clinicians and colorectal cancer screening programme organisers need to know. Gut 64(8):1327-1337

8. Soares-Weiser KBJ, Duffy S, St John J, Smith A, Westwood M, Kleijnen J (2007) Diagnostic accuracy and cost-effectiveness of faecal occult blood test (FOBT) used in screening for colorectal cancer: a systematic revew. The University of York, York

9. Logan RF, Patnick J, Nickerson C, Coleman L, Rutter MD, von Wagner C (2012) Outcomes of the Bowel Cancer Screening Programme (BCSP) in England after the first 1 million tests. Gut 61(10):1439-1446

10. Probert CS, Reade S, Ahmed I (2014) Fecal volatile organic compounds: a novel, cheaper method of diagnosing inflammatory bowel disease? Expert Rev Clin Immunol 10(9):1129-1131

11. Kataoka H, Saito K, Kato H, Masuda K (2013) Noninvasive analysis of volatile biomarkers in human emanations for health and early disease diagnosis. Bioanalysis 5(11):1443-1459

12. Arasaradnam RP, McFarlane MJ, Ryan-Fisher C, Westenbrink E, Hodges P, Thomas MG et al (2014) Detection of colorectal cancer (CRC) by urinary volatile organic compound analysis. PLoS One 9(9): e108750

13. Westenbrink E, Arasaradnam RP, O'Connell N, Bailey C, Nwokolo C, Bardhan KD et al (2015) Development and application of a new electronic nose instrument for the detection of colorectal cancer. Biosens Bioelectron 67:733-738

14. Di Lena M, Porcelli F, Altomare DF (2016) Volatile Organic Compounds (VOC) as new biomarkers for colorectal cancer: a review. Colorectal Dis 18(7):654-663

15. Jobu K, Sun C, Yoshioka S, Yokota J, Onogawa M, Kawada C et al (2012) Metabolomics study on the biochemical profiles of odor elements in urine of human with bladder cancer. Biol Pharm Bull 35(4):639-642

16. Silva CL, Passos M, Camara JS (2012) Solid phase microextraction, mass spectrometry and metabolomic approaches for detection of potential urinary cancer biomarkers-a powerful strategy for breast cancer diagnosis. Talanta 89:360-368

17. Van de Schee M (2015) The volatile metabolome and microbiome in pulmonary and gastro-intestinal disease. Amsterdam, Holland

18. Bhatt A, Parsi MA, Stevens T, Gabbard S, Kumaravel A, Jang S et al (2016) Volatile organic compounds in plasma for the diagnosis of esophageal adenocarcinoma: a pilot study. Gastrointest Endosc 84(4):597-603
19. Capelli L, Taverna G, Bellini A, Eusebio L, Buffi N, Lazzeri M et al (2016) Application and uses of electronic noses for clinical diagnosis on urine samples: a review. Sensors (Basel) 16(10):1708

20. Arasaradnam RP, McFarlane M, Daulton E, Skinner J, O'Connell $\mathrm{N}$, Wurie $\mathrm{S}$ et al (2016) Non-invasive exhaled volatile organic biomarker analysis to detect inflammatory bowel disease (IBD). Dig Liver Dis 48(2):148-153

21. Covington JA, van der Schee MP, Edge AS, Boyle B, Savage RS, Arasaradnam RP (2015) The application of FAIMS gas analysis in medical diagnostics. Analyst 140(20):6775-6781

22. Covington JA, Wedlake L, Andreyev J, Ouaret N, Thomas MG, Nwokolo CU et al (2012) The detection of patients at risk of gastrointestinal toxicity during pelvic radiotherapy by electronic nose and FAIMS: a pilot study. Sensors (Basel) 12(10):13002-13018

23. Arasaradnam RP, Westenbrink E, McFarlane MJ, Harbord R, Chambers S, O'Connell N et al (2014) Differentiating coeliac disease from irritable bowel syndrome by urinary volatile organic compound analysis-a pilot study. PLoS One 9(10):e107312

24. Chawla NVBK, Hall LO, Kegelmeyer WP (2002) SMOTE: synthetic minority over-sampling technique. J Artif Intell 16:321-357

25. Cairns SR, Scholefield JH, Steele RJ, Dunlop MG, Thomas HJ, Evans GD et al (2010) Guidelines for colorectal cancer screening and surveillance in moderate and high risk groups (update from 2002). Gut 59(5):666-689

26. Bouvard V, Loomis D, Guyton KZ, Grosse Y, Ghissassi FE, Benbrahim-Tallaa L et al (2015) Carcinogenicity of consumption of red and processed meat. Lancet Oncol 16(16):1599-1600

27. Altomare DF, Di Lena M, Porcelli F, Trizio L, Travaglio E, Tutino $M$ et al (2013) Exhaled volatile organic compounds identify patients with colorectal cancer. Br J Surg 100(1):144-150

28. Haick H, Broza YY, Mochalski P, Ruzsanyi V, Amann A (2014) Assessment, origin, and implementation of breath volatile cancer markers. Chem Soc Rev 43(5):1423-1449

29. van de Kant KD, van der Sande LJ, Jobsis Q, van Schayck OC, Dompeling E (2012) Clinical use of exhaled volatile organic compounds in pulmonary diseases: a systematic review. Respir Res 13:117

30. Batty CA, Cauchi M, Lourenco C, Hunter JO, Turner C (2015) Use of the analysis of the volatile faecal metabolome in screening for colorectal cancer. PLoS One 10(6):e0130301

31. de Meij TG, Larbi IB, van der Schee MP, Lentferink YE, Paff T, Terhaar Sive Droste JS et al (2014) Electronic nose can discriminate colorectal carcinoma and advanced adenomas by fecal volatile biomarker analysis: proof of principle study. Int J Cancer 134(5):1132-1138

32. Amal H, Leja M, Funka K, Lasina I, Skapars R, Sivins A et al (2016) Breath testing as potential colorectal cancer screening tool. Int J Cancer 138(1):229-236

33. Altomare DF, Porcelli F, Picciariello A, Pinto M, Di Lena M, Caputi Iambrenghi O, Ugenti I, Guglielmi A, Vincenti L, De Gennaro $G$ (2016) The use of the PEN3 e-nose in the screening of colorectal cancer and polyps. Tech Coloproctol 20:405-409. https ://doi.org/10.1007/s10151-016-1457-z

34. Hoff G, Sauar J, Vatn MH, Larsen S, Langmark F, Moen IE et al (1996) Polypectomy of adenomas in the prevention of colorectal cancer: 10 years' follow-up of the Telemark Polyp Study I. A prospective, controlled population study. Scand J Gastroenterol 31(10):1006-1010

35. Atkin WS, Morson BC, Cuzick J (1992) Long-term risk of colorectal cancer after excision of rectosigmoid adenomas. N Engl J Med 326(10):658-662

36. O'Brien MJ, Winawer SJ, Zauber AG, Gottlieb LS, Sternberg SS, Diaz B et al (1990) The national polyp study. Patient and polyp characteristics associated with high-grade dysplasia in colorectal adenomas. Gastroenterology 98(2):371-379 
37. Rees CJ, Gibson T, Rutter MD, Baragwanath P, Pullan R, Feeney $M$ et al (2016) UK key performance indicators and quality assurance standards for colonoscopy. Gut 65(12):1923-1929

38. Mackie A (2015) Moving from guaiac faecal occult blood test (gFOBT) to a faecal immunochemical test for haemoglobin (FIT) in the bowel screening programme: a consultation. London. Available at: https://legacyscreening.phe.org.uk/policydb_download. php?doc $=802$. Accessed 3 July 2018

39. Allers M, Langejuergen J, Gaida A, Holz O, Schuchardt S, Hohlfeld JM et al (2016) Measurement of exhaled volatile organic compounds from patients with chronic obstructive pulmonary disease (COPD) using closed gas loop GC-IMS and GC-APCI-MS. J Breath Res 10(2):026004
40. Mochalski P, Wiesenhofer H, Allers M, Zimmermann S, Guntner AT, Pineau NJ et al (2018) Monitoring of selected skinand breath-borne volatile organic compounds emitted from the human body using gas chromatography ion mobility spectrometry (GC-IMS). J Chromatogr B Analyt Technol Biomed Life Sci 1076:29-34

41. Lawler M, Alsina D, Adams RA, Anderson AS, Brown G, Fearnhead NS et al (2018) Critical research gaps and recommendations to inform research prioritisation for more effective prevention and improved outcomes in colorectal cancer. Gut 67(1):179-193

Publisher's Note Springer Nature remains neutral with regard to jurisdictional claims in published maps and institutional affiliations. 